\title{
A Parametric Ribcage Geometry Model Accounting for Variations among the Adult Population
}

\author{
Yulong Wang ${ }^{1,2}$ \\ Libo Cao ${ }^{1}$ \\ Zhonghao Bai ${ }^{1}$ \\ Matthew P. Reed ${ }^{2,3}$ \\ Jonathan D. Rupp $2,4,5$ \\ Carrie N. Hoff ${ }^{6}$ \\ Jingwen $\mathrm{Hu}^{2,7}$ \\ ${ }^{1}$ State Key Laboratory of Advanced Design and Manufacturing for Vehicle Body, Hunan \\ University, Changsha, Hunan, China \\ ${ }^{2}$ University of Michigan Transportation Research Institute, Ann Arbor, MI, USA \\ ${ }^{3}$ Center for Ergonomics, Industrial and Operations Engineering, University of Michigan, \\ Ann Arbor, MI, USA \\ ${ }^{4}$ Department of Biomedical Engineering, University of Michigan, Ann Arbor, MI, USA \\ ${ }^{5}$ Department of Emergency Medicine, University of Michigan, Ann Arbor, MI, USA \\ ${ }^{6}$ Department of Radiology, University of Michigan, Ann Arbor, MI, USA \\ ${ }^{7}$ Department of Mechanical Engineering, University of Michigan, Ann Arbor, MI, USA
}

\section{Corresponding Author:}

Jingwen $\mathrm{Hu}, \mathrm{PhD}$

University of Michigan Transportation Research Institute

2901 Baxter Rd,

Ann Arbor, MI, 48109, USA

Tel: 734-763-6398

Fax: 734-647-3330

Email: jwhu@umich.edu

Keywords: Ribcage, Parametric model, Morphological variation, Human characteristics, Adult population

Word Count: 4010 
The objective of this study is to develop a parametric ribcage model that can account for morphological variations among the adult population. Ribcage geometries, including 12 pair of ribs, sternum, and thoracic spine, were collected from CT scans of 101 adult subjects through image segmentation, landmark identification (1,016 for each subject), symmetry adjustment, and template mesh mapping (26 180 elements for each subject). Generalized Procrustes analysis (GPA), principal component analysis (PCA), and regression analysis were used to develop a parametric ribcage model, which can predict nodal locations of the template mesh according to age, sex, height, and body mass index (BMI). Two regression models, a quadratic model for estimating the ribcage size and a linear model for estimating the ribcage shape, were developed. The results showed that the ribcage size was dominated by the height $(\mathrm{p}=0.000)$

11 and age-sex-interaction $(\mathrm{p}=0.007)$ and the ribcage shape was significantly affected by the age $(\mathrm{p}=0.0005)$, 12 sex $(\mathrm{p}=0.0002)$, height $(\mathrm{p}=0.0064)$ and $\mathrm{BMI}(\mathrm{p}=0.0000)$. Along with proper assignment of cortical bone 13 thickness, material properties and failure properties, this parametric ribcage model can directly serve as 14 the mesh of finite element ribcage models for quantifying effects of human characteristics on thoracic 15 injury risks.

\section{Introduction}

17 Crash injury data analyses have shown that thoracic injuries are the second leading cause of fatalities and severe injuries in motor vehicle crashes (MVCs), and the risks of thoracic injuries are significantly associated with occupant characteristics, such as age, sex, height, and body mass index (BMI), a parameter measuring the obesity level.

21 As age increases, the human thorax experiences both morphological changes and material property 22 changes (Gayzik et al., 2006; Gayzik et al., 2008; Kent et al., 2005). Field data analyses, computer 23 simulations and cadaver tests have all shown that the incidence of AIS 2+ thoracic injuries increased with 24 age (Carter et al., 2014; Ridella et al., 2012; Zhou et al., 1996). The risk of ribcage injuries in crashes also 

et al., 2005; Kindig et al., 2010). In particular, the risk of being seriously injured in crashes is higher for women than men (Bose et al., 2011; Parenteau et al., 2013), and the increase of thoracic injury risk with age is greater for women than men (Carter et al., 2014; Parenteau et al., 2013; Ridella et al., 2012). In addition, obese occupants are at increased risk of thoracic injuries compared with non-obese (Cormier, 2008; Forman et al., 2009a; Forman et al., 2009b; Shi et al., 2015; Turkovich, 2011).

Morphological variations in the human ribcage, such as the shape, size and cortical bone thickness, as well as the material and failure properties of the ribcage, are expected to affect the impact response and injury tolerance of the thorax, especially among vulnerable populations such as the elderly, women, and the obese. These variations in ribcage geometry could be evaluated through statistical shape analysis

11 (SSA), a common technique to assess the size and shape variations in human skeleton and organs, such as femur (Bredbenner and Nicolella, 2008; Bryan et al., 2009; Zhu and Li, 2011), tibia (Baka et al., 2014; Bredbenner et al., 2010), liver (Lamecker et al., 2004; Lu and Untaroiu, 2014) and spleen (Yates et al., 2016). Gayzik et al. (2008) quantified the age-related shape change of human ribcage using rib landmarks through morphometric and multivariate regressions. Weaver et al. (2014a; 2014b) analyzed a larger set of landmarks on the ribcage obtained from analysis of CT scans from 339 subjects aged 0-100 years. The

17 size and shape of all the ribs and sternum were significantly affected by age in both male and female. Based on CT scans from 89 adult subjects, Shi et al. (2014) developed a statistical model to predict ribcage geometry accounting for age, sex, height and BMI using principal component analysis (PCA) and 20 regression.

21 To improve the model reported by Shi et al. (2014) and allow the ribcage geometry model to be used 22 directly for assessing the effect of morphological variation on chest injury risk, the objective of this study 23 is to build a parametric ribcage model accounting for the age, sex, height, and BMI effects on the ribcage 24 morphology, including all 24 ribs, sternum, and thoracic spine. 
1

2

3

4

5

6

7

8

9

10

11

12

13

14

15

16

17

18

19

20

21

22

23

\section{Methods}

Anonymous clinical ribcage CT scans $(\mathrm{n}=101)$ were obtained from University of Michigan Health System using a protocol approved by an institutional review board at the University of Michigan. All subjects were adult female $(n=47)$ or male $(n=54)$ patients without skeletal pathology. The age, sex, height and BMI distributions of the subjects are shown in Figure 1. Except the high correlation between the height and sex, no significant correlation was found among these four parameters.

\section{[Figure 1]}

Figure 2 shows the overview of the method for developing the parametric ribcage model accounting for morphological variations among the adult population. First, the ribcage geometry for each subject was collected through a series of image analyses, including threshold-based image segmentation, landmark identification on each rib, sternum and spine, landmark re-processing through B-spline, and landmark symmetry adjustment. After the landmarks were identified, a template ribcage finite element (FE) model from THUMS (Total Human Model for Safety) version 4.01(Hayashi et al., 2008; Iwamoto et al., 2002) was morphed into the geometry of each subject using a mesh morphing method. The ribcage model of THUMS, including 24 ribs, sternum and the thoracic spine, consists of 26,180 shell elements and 26,139 nodes. Generalized Procrustes analysis (GPA), PCA, and regression analyses were then used to develop a parametric model that used age, height, BMI and sex to predict nodal locations of the FE ribcage mesh.

[Figure 2]

\subsection{Landmark identification}

All the CT data were collected using a resolution of $512 \times 512$ pixels with $1.25 \mathrm{~mm}$ between slices. Selected scans were imported into Mimics (Materialise, Plymouth, MI) for image segmentation. A semiautomated threshold method was used to segment the thoracic skeleton, including the 24 ribs, the sternum, and the thoracic vertebrae from the CT scans. 
Landmarks were collected at ten cross-sections along each of the 24 ribs for each subject. Four landmarks were identified on each cross-section following the method used by Shi et al. (2014). To ensure the crosssections being evenly distributed along the rib, B-spline interpolation was used to generate curves along the rib top and bottom ridges, and these curves were divided equally into nine segments with ten crosssections. As a result, 960(40x24) landmarks were identified on the ribs. For the sternum, a pair of landmarks were identified at each sternocostal joint and sternoclavicular joint, which resulted in 32 landmarks on the sternum. Two landmarks were identified at the tips of the spinal process on each of the T1-T12 vertebrae. As a result, a total of 1,016 landmarks on the ribcage, including 960 landmarks on the ribs, 32 landmarks on the sternum and 24 landmarks on the spine, were identified for each subject. Figure 2 shows an example of identified landmarks on the ribs, sternum, and thoracic spine.

\subsection{Spine lateral curvature adjustment}

12 In general, the landmarks identified from each subject were not perfectly symmetric due to the spine lateral curvature and the biological difference between the two sides of the ribs. Relative speaking, the spine lateral curvature generated much greater differences between the left and right sides of the ribcage than the biological differences between the corresponding ribs on the two sides. The spine lateral curvature also varied significantly among subjects, which introduced errors/variations that are unrelated

17 to the subject age, sex, stature, and BMI. Therefore, in this study, we adjusted the spine lateral curvature, so that the two sides of the ribcage are symmetric in terms of the rib positions. In addition, with the goal of building parametric FE models, a symmetric model was also developed for each subject by averaging the rib geometries from the left and the right. The steps for adjusting the spine lateral curvature and

21 ribcage symmetry are as follows. First, a global center plane of the ribcage for each subject was estimated

22 using a least-square method based on the landmarks. All landmarks of each subject were rotated rigidly so 23 that the estimated global center plane was aligned to the $\mathrm{X}-\mathrm{Z}$ plane. Second, a local center plane was 24 estimated for each pair of ribs. A reflection was performed for the landmarks on each pair of ribs around 25 the local center plane, and the average locations between the original and the reflected landmarks were 
1 considered as the final landmark locations. Lastly, the local center plane of each vertebra was laterally

2 translated to match the global center plane, so that the whole thoracic spine can be adjusted into the center.

3 In this process, the distances between the adjacent vertebrae were maintained, so that the height of the

$4 \quad$ thoracic spine was not affected.

\section{$5 \quad 2.3$ Mesh mapping}

6 In this study RBF-TPS (Radial Basis Function with Thin-Plate Spline) was used to morph the template

7 ribcage FE mesh to the ribcage geometry for each subject. To do this, the same set of landmarks was

8 identified on the template FE mesh, and mesh morphing was then conducted by the RBF-TPS, so that the

9 morphed mesh matched the geometry defined by the target landmarks. Figure 2 shows the template mesh

10 with the source landmarks and the morphed mesh with the target landmarks. Details about the RBF-TPS

11 have been previous described by (Donato and Belongie, 2002). Similar methods have been used to build

12 parametric FE models previously (Klein et al., 2015; Ma et al., 2011; Wang et al., 2015).

\section{$13 \quad 2.4$ Generalized Procrustes Analysis}

14 GPA was used to align the ribcages from different subjects and separate the ribcage size and the shape 15 variations. GPA has been widely used in morphometric studies of anthropology and anatomy 16 (Dijksterhuis and Gower, 1992; Slice, 2005; Stegmann and Gomez, 2002). In this study, two types of 17 GPAs were performed, one on the entire set of ribcage landmarks and the other on each rib individually, so that the variations on each rib and the entire ribcage can be analyzed separately. The steps of these two

19 types of GPAs are the same, which included:

20 1. Construct matrix $x_{i}$ with a dimension of $\mathrm{n} \times 3$ to represent ribcage shape for each subject, in which $\mathrm{n}$ is

21 the landmark number and each row of the matrix represents the landmark coordinate.

22 2. Calculate the mean shape $\bar{x}$ of all the subjects $x_{i}$; 
$\bar{x}=\frac{1}{m} \sum_{i=1}^{m} x_{i}$

13 . Use the Procrustes superimposition to align the remaining shapes to the mean shape;

$x_{i}^{\prime}=\frac{1}{C S}\left(x_{i}-C\right) T$

2 Where $T$ is a $3 \times 3$ matrix that includes the orthogonal rotation and reflection components, $C$ is the

3 translation component along $\mathrm{x}, \mathrm{y} \mathrm{z}$ direction, and $C S$ is the centroid size, the square root of the sum of

4 squared coordinate values in all dimensions as shown in Equation 3.

$C S=\sqrt{\operatorname{tr}\left(x_{i}^{T} x_{i}\right)}$

5 Where "tr" is the trace of $x_{i}^{T} x_{i}$, a matrix with dimension of $3 \times 3$.

64 . Re-calculate the mean shape $\bar{x}^{\prime}$ of all the aligned shapes $x_{i}^{\prime}$;

$\bar{x}^{\prime}=\frac{1}{m} \sum_{i=1}^{m} x_{i}^{\prime}$

7 5. If the difference between $\bar{x}$ and $\bar{x}^{\prime}$ is greater than the threshold (1e-6 mm), return to step 3.

8 After the full GPA, the ribcage geometry of each subject was separated into two components, the centroid

$9 \quad$ size $(C S)$ and the shape vector $\left(x_{i}-C\right) T$.

\subsection{Parametric ribcage model}

11 To separate the size and shape effects, two regression models were developed, including a quadratic

12 model for quantifying the size effect and a linear model for quantifying the shape effect.

13 A stepwise regression method was used to build a quadratic model for estimating the ribcage size (CS), in

14 which only the significant input variables was selected in the final model. The input parameters used in 15 the regression model for $C S$ were age, height, BMI, sex, and their first-order interactions. 
1 To build a regression model to predict ribcage shape, PCA was first used to quantify the primary modes

2 of variation in the dataset and to express the dataset as a set of new orthogonal variables called principal

3 components (PCs), which can be more readily analyzed when the data with a high number of dimensions

4 (Abdi and Williams, 2010; Allen et al., 2003). Assuming that there are $m$ subjects and the shape vector for

5 each subject, composed of the vector of coordinates of all landmarks, is $g_{i}$, the centered shape matrix can

6 be expressed as $G=\left[g_{1}-\bar{g}, g_{2}-\bar{g}, g_{3}-\bar{g} \ldots g_{m}-\bar{g}\right]_{3 n \times m}$, in which $\bar{g}$ is the mean shape vector.

7 Using PCA, $G$ can be decomposed as $G=P_{k} * S_{k}+\varepsilon$, where $S_{k}$ is the first $k$ PCs of the first $k$ eigenvectors of

$8 \mathrm{G}^{*} \mathrm{G}^{\prime}$, and $P_{k}$ is the first $k$ PC scores. Since $S_{k} * S_{k}^{T}=I, P_{k}$ can be expressed as

$9 \quad P_{k} \approx G * S_{k}^{T}$

10 Set $F=[$ Age,Sex,Height,BMI,1]', in which each column represents parameters from a subject. Using a

11 linear regression model, $P_{k}$ can be expressed as a function of the age, sex, height and BMI.

$12 \quad P_{k}=M * F+\varepsilon$

13 in which each row of $M$ represents the coefficients of the linear regression model for the PC scores. The

14 coefficient matrix $M$ can be estimated using least-square method by taking the Moore-Penrose pseudo 15 inverse $\mathrm{F}^{+}$.

$16 \quad M=\left(P_{k}-\varepsilon\right) F^{+} \approx P_{k} F^{+}$

17 According to the PCA and the regression model of the centroid size, a full set of ribcage landmarks can be 18 predicted from age, sex, height and BMI using Equation 8:

$19 g_{\text {Pre }}=\left[\left(F_{1 \times 5} * M_{5 \times k}\right) * S_{k}+\bar{g}\right] * C S$

203 Results

$21 \quad 3.1$ Generalized Procrustes Analysis 
1 The relationships between the centroid size $(C S)$ in GPA and the age, sex, height and BMI are shown in

2 Figure 3. CS and height were strongly correlated, but age and BMI had near-zero correlation with CS.

3 Based on the results from the stepwise quadratic regression, $C S$ was only significantly associated with the

4 height $(\mathrm{p}=0.000)$ and age-sex interaction $(\mathrm{p}=0.007)$. The final regression model is:

$5 \quad C S=7853.4 *$ Height $+19.1 * \operatorname{Sex} *$ Age $+6895.4 \quad\left(\mathrm{R}^{2}=0.771, \mathrm{p}=0.000\right)$

[Figure 3]

\section{$7 \quad 3.2$ Parametric ribcage model}

8 In our study, 60 PC were retained from the PCA, which accounted for $99 \%$ of the variance. The

9 percentages of variance accounted by the first 5 PCs were $41.2 \%, 11.6 \%, 8.5 \%, 6.0 \%$ and $5.2 \%$,

10 respectively. The significance of the regression model for ribcage shape was assessed through

11 permutation test (Weaver et al., 2014a). The results showed that the ribcage shape was affected

12 significantly by the age $(\mathrm{p}=0.0005)$, sex $(\mathrm{p}=0.0002)$, height $(\mathrm{p}=0.0064)$ and BMI $(\mathrm{p}=0.0000)$. Age, sex,

13 height, and BMI all had significant effects on PC1. Age also had significant effects on PC2 and PC4. BMI

14 had significant effects on PC2 and PC5.

15 By combining the regression models for the ribcage size and shape, the ribcage geometry with any given 16 age, sex, height, and BMI can be predicted using Equation 10:

$17 \quad M_{P r e}=\left[\left(F_{1 \times 5} * M_{5 \times 60}\right) * S_{60 \times 3 n}+\bar{g}_{1 \times 3 n}\right] *(7853.4 *$ Height $+19.1 *$ Sex $*$ Age +6895.4$)$

where, $F_{1 \times 5}=\left[\right.$ Age,Sex,Height,BMI,1], $\mathrm{M}_{5 \times 60}$ is the coefficient matrix for the first $60 \mathrm{PCs}, \mathrm{S}_{60 \times 3 \mathrm{n}}$ is the matrix of first $60 \mathrm{PCs}$, and $\bar{g}_{1 \times 3 n}$ is the mean of the shape matrix.

Figure 5 shows the ribcages with different age, sex, height and BMI predicted by the parametric model. As shown in Figure 5(a), with the same BMI and height, the predicted ribcage of an 80 year-old man is wider and deeper than a 20 year-old man. Figure 5(b) shows that even with the same age, height, and BMI, the ribcage of male is generally wider and deeper than female. The effect of height on the ribcage size is 
1 obvious as shown in Figure 5(c). Figure 5(d) shows that BMI had a larger effect on the lower portion of

2 the ribcage than the upper portion. Specifically, the breadth of the lower portion of the ribcage is much

3 larger than the upper portion with a higher BMI, while no significant difference was observed in terms of

4 the breadth of the upper and lower portions of the ribcage with a normal BMI.

[Figure 4]

\section{$6 \quad 3.3$ Model error analysis}

7 The coefficient of determination $\mathrm{R}^{2}$ values for the parametric model were calculated through Equation 9 ,

8 where $S S_{\text {res }}$ is the sum of squared errors between the observed and predicted landmark coordinates, and

$9 \quad S S_{t o t}$ is the sum of squared differences between the average and predicted landmark coordinates. The $\mathrm{R}^{2}$

10 value for the ribcage size model and the ribcage shape model were 0.77 and 0.56 , respectively, and the

11 overall $\mathrm{R}^{2}$ value for the parametric ribcage model was 0.51 .

$12 \mathrm{R}^{2}=1-\frac{\mathrm{SS}_{\mathrm{res}}}{\mathrm{SS}_{\mathrm{tot}}}$

13 To evaluate the model accuracy, Euclidean distance was first calculated for every node on the ribcage

14 between the symmetry-adjusted landmark locations and the model prediction. Two types of average errors,

15 one for single ribs and one for the whole ribcage, were calculated. The average error for a single rib only

16 considered the shape/size difference but not the location difference between the model-predicted

17 geometry and the true subject geometry, while the average error for the whole ribcage considered both the

18 shape/size difference and location difference for all the ribs.

19 The average errors for single ribs were generally around 3 to $4 \mathrm{~mm}$. Figure 5(a) shows the average error

20 distribution of the 8 th rib for all the 101 subjects. No significant correlation between the average error and

21 the input variables (age, height, and BMI) was found. Figure 5(b) shows the average errors for the whole

22 ribcage of all 101 subjects with age, height and BMI. Errors were similar across values of predictors.

[Figure 5] 


\section{1}

\section{Discussion}

2

\subsection{Error analysis}

3 Several reasons may contribute to the geometry errors in our current parametric ribcage model. First, as

4 shown in Figure 6, the average errors for single ribs (Figure 6a) were much lower than those for the whole

5 ribcage (Figure 6b). It indicated that the errors were mainly caused by the rib locations rather than the

6 shapes of single ribs. Moreover, the two floating ribs had the largest errors, especially for the $12^{\text {th }}$ rib.

7 Second, large variations existed among the selected subjects, which affected both the size and the shape

8 of the ribcage geometry. Since the parametric ribcage model was separated into size regression model and

9 shape regression model in this study, the errors for the size and shape models can be quantified separately

10 (Figures 6a and b). In general, the size errors were smaller than the shape errors, which was consistent

11 with the $\mathrm{R}^{2}$ values reported previously. Figure 6(c) showed the total error distributions and the error

12 distributions caused by the size only for three subjects, including a small female, a mid-size male and a

13 large male. It showed consistent findings as shown in Figure 6(a) and 6(b). Third, the current parametric

14 model only included age, sex, height, and BMI as the predictors. Other input variables may be needed to

15 further improve the accuracy of the model. Third, the current parametric model only included age, sex,

16 height, and BMI as the predictors. Other input variables, such as the shoulder breadth, chest breadth,

17 depth and circumference, may improve the model capability of accounting for the ribcage geometry

18 variations. However, such parameters are not always available for a specific subject without measuring

19 them. Therefore, the benefit of including those parameters might be limited. Lastly, a linear regression

20 model was used for analyzing the ribcage shape. Other nonlinear models may potentially reduce the error,

21 but it needs further investigation.

22

\section{$23 \quad 4.2$ Morphologic effects from human characteristics}


Many previous studies have investigated the effects of human characteristics on ribcage geometry. Kent et al.(2005) used ribcage CT scans from 161 subjects ranged from 18 to 89 years old to quantify the rib

3 angles in the sagittal plane. Using the same rib angle definition of the $9^{\text {th }}$ rib, the angle between the rib 4 and the longitudinal direction of the torso, the parametric model developed in the current study predicted 5 that older, more obese, and male subjects have greater rib angles compared to younger, leaner, and female 6 subjects. In particular, every 1-year-old increase in age and $1-\mathrm{kg} / \mathrm{m}^{2}$ increase in BMI were associated with 7 an average of $0.06^{\circ}$ and $0.45^{\circ}$ increase in the angle, respectively, which is consistent to the corresponding 8 rib angle increases $\left(0.0412^{\circ}\right.$ and $\left.0.572^{\circ}\right)$ reported by Kent et al. (2005). Our model also predicted that 9 male subjects had an average of $3.77^{\circ}$ greater rib angle than female, while the corresponding angle increase reported by Kent et al. (2005) was $1.03^{\circ}$.

11 Figure 7 (a) shows the thoracic spine curvatures of a 20 year-old and 80 year-old man with $175 \mathrm{~cm}$ height 12 and $25 \mathrm{~kg} / \mathrm{m}^{2}$ BMI predicted by our model and those reported by Gayzik et al. (2008). Despite the difference in landmark selections, both studies show increased kyphosis with the increase in age.

15 Even though the current study shared a subset $(n=45)$ of the sampled subjects and used similar statistical methods as those in Shi et al. (2014), several major improvements have been made. Firstly, the current 17 study collected landmarks on all 24 ribs, the sternum and the thoracic spine, while the previous study only considered the left side of the ribs. Secondly, in the current study, the lateral curvature of the thoracic spine was corrected for each subject, so that all the ribcages were registered into the same initial posture 20 for statistical analysis. Thirdly, two regression models, a size model and a shape model, were developed 21 in the current study, which was critical for quantifying the effect of human characteristics on the ribcage 22 size and shape separately. Finally, the THUMS ribcage mesh was morphed into the geometries from all 23 the subjects, so that a detailed ribcage FE mesh can be rapidly generated using age, sex, stature, and BMI 24 based on the statistical model. This mesh can be directly used for FE simulations, along with the proper 25 assignment of the ribcage cortical bone thickness and material properties. 
1 Morphometric variations in the ribs and sternum for both children and adults were investigated by

2 Weaver et al. (2014a; 2014b). However, the size and shape variations of the ribcage geometry may be

3 significantly affected by the growing effects in children, which made it challenging for a single regression

4 model to capture variations for both child and adult populations. In our study, we only focused on the

5 adult population, but both studies found similar size and shape of the sternum for the adults aged 30-100

6 years with a given stature and sex. Weaver et al. (2014a; 2014b) also found that the ribcage experienced

7 increased thoracic kyphosis and superior rotation of the ribs with increased age, consistent with the

8 current study. In addition, the current study collected landmarks on the thoracic spine and used a template

9 mesh directly from an FE human model for building the parametric model, which have not been reported

10 in previous studies.

\section{$11 \quad 4.3$ Limitations and future work}

12 A major limitation of this study is that the cortical bone thickness could not be accurately measured since

13 the resolution of the CT scans was not sufficient to quantify the small thickness values. In this study,

14 although the cortical bone thickness is not available, the diameter and the total cross-sectional area of the

15 rib can be readily used for developing ribcage FE models. Future work is necessary to use higher-

16 resolution imaging techniques (such as micro-CT) to extract the cortical bone thickness of the ribs. The

17 current model also does not capture inter-subject variation in the cross-section shape, since only four

18 landmarks were obtained from each cross section.

19 The landmarks were manually collected on the ribcage, spine and sternum. This process is time20 consuming and the repeatability of the landmark extraction may vary across individuals. However, the 21 mesh morphing/mapping method enabled the same set of mesh to be evenly distributed throughout the 22 ribs, hence improved the consistency of geometry extraction.

23 To assess the effect of the human characteristics on the thorax injury risk, computational modeling is a 24 powerful and versatile tool. Previous studies (El-Jawahri et al., 2010; Ito et al., 2009) have developed age- 
1 dependent FE human thorax models to investigate the age effects on thorax impact responses. However,

2 those studies generally only changed the angle of the ribs, but did not considered detailed morphologic

3 changes on the ribcage due to aging. The parametric ribcage geometry model developed in this study used

4 the THUMS mesh as the template mesh; therefore, along with proper assignment of cortical bone

5 thickness, material properties and failure properties, it can be used as the FE mesh for thoracic injury

6 prediction.

\section{5. Conclusions}

8 This study developed a parametric ribcage model accounting for the effects of age, sex, height, and BMI

9 on ribcage morphology. The ribcage geometries were collected from CT scans of 101 adult subjects

10 through a series of image processing, landmarks identification, and landmark symmetry adjustment. A

11 template FE ribcage model was morphed into geometry from each subject using mesh morphing/mapping

12 method. GPA, PCA, and regression analyses were then used to develop a parametric ribcage model,

13 which can predict an FE mesh based on age, sex, height, and BMI. Two regression models, a quadratic

14 model for estimating the ribcage size and a linear model for estimating the ribcage shape, were developed.

15 The results showed that the ribcage size was dominated by the height and age-sex-interaction, and the

16 ribcage shape was significantly affected by the age, sex, height and BMI. Along with proper assignment

17 of cortical bone thickness, material properties and failure properties, this parametric ribcage model can

18 directly serve as the mesh of FE ribcage models for quantifying effects of human characteristics on 19 thoracic injury risks.

\section{Acknowledgement}

21 This study was supported by National Science Foundation (Award No: 1300815), National Natural

22 Science Foundation of China (Award No: 51475153), State Key Laboratory of Advanced Design and

23 Manufacturing for Vehicle Body (Award No: 71575004) and a grant from China Scholarship Council. 
1 The authors would like to thank Alyssa Blunt and Marcellus Shantz for their contributions to image

2 segmentation and landmark identification.

\section{$5 \quad$ References}

6 Abdi, H., Williams, L.J., 2010. Principal component analysis. Wiley Interdisciplinary Reviews:

7 Computational Statistics 2, 433-459.

8 Allen, B., Curless, B., Popović, Z., 2003. The space of human body shapes: reconstruction and

9 parameterization from range scans. In ACM transactions on graphics (TOG) 22(3), 587-594.

10 Baka, N., Kaptein, B.L., Giphart, J.E., Staring, M., de Bruijne, M., Lelieveldt, B.P., Valstar, E., 2014.

11 Evaluation of automated statistical shape model based knee kinematics from biplane fluoroscopy. Journal

12 of biomechanics $47,122-129$.

13 Bellemare, F., Jeanneret, A., Couture, J., 2003. Sex differences in thoracic dimensions and configuration.

14 American journal of respiratory and critical care medicine 168, 305-312.

15 Bose, D., Segui-Gomez, M., Crandall, J.R., 2011. Vulnerability of female drivers involved in motor 16 vehicle crashes: an analysis of US population at risk. American journal of public health 101, 2368.

17 Bredbenner, T.L., Eliason, T.D., Potter, R.S., Mason, R.L., Havill, L.M., Nicolella, D.P., 2010. Statistical 18 shape modeling describes variation in tibia and femur surface geometry between Control and Incidence 19 groups from the osteoarthritis initiative database. Journal of biomechanics 43, 1780-1786.

20 Bredbenner, T.L., Nicolella, D.P., 2008. Statistical shape and density based finite element modeling of the 21 human proximal femur. Journal of Biomechanics 27, 1159-1168.

22 Bryan, R., Nair, P.B., Taylor, M., 2009. Use of a statistical model of the whole femur in a large scale, 23 multi-model study of femoral neck fracture risk. Journal of biomechanics 42, 2171-2176. 
1 Carter, P.M., Flannagan, C.A., Reed, M.P., Cunningham, R.M., Rupp, J.D., 2014. Comparing the effects

2 of age, BMI and gender on severe injury (AIS 3+) in motor-vehicle crashes. Accident Analysis \&

3 Prevention 72, 146-160.

4 Cerney, M.M., Adams, D.C., 2004. Sequestering size: The role of allometry and gender in digital human

5 modeling. SAE Transactions Journal of Aerospace 113, 208-214.

6 Cormier, J.M., 2008. The influence of body mass index on thoracic injuries in frontal impacts. Accident

$7 \quad$ Analysis \& Prevention 40, 610-615.

8 Dijksterhuis, G.B., Gower, J.C., 1992. The interpretation of generalized procrustes analysis and allied

9 methods. Food Quality and Preference 3, 67-87.

10 Donato, G., Belongie, S., 2002. Approximate thin plate spline mappings, Computer Vision—ECCV 2002.

11 Springer, pp. 21-31.

12 El-Jawahri, R.E., Laituri, T.R., Ruan, J.S., Rouhana, S.W., Barbat, S.D., 2010. Development and

13 validation of age-dependent FE human models of a mid-sized male thorax. Stapp car crash journal 54, 14407.

15 Forman, J., Lopez-Valdes, F., Lessley, D., Kindig, M., Kent, R., Ridella, S., Bostrom, O., 2009a. Rear 16 seat occupant safety: an investigation of a progressive force-limiting, pretensioning 3-point belt system

17 using adult PMHS in frontal sled tests. Stapp car crash journal 53, 49.

18 Forman, J., Lopez-Valdes, F.J., Lessley, D., Kindig, M., Kent, R., Bostrom, O., Year The effect of obesity

19 on the restraint of automobile occupants. In Annals of Advances in Automotive Medicine/Annual

20 Scientific Conference.

21 Gayzik, F.S., Loftis, K.L., Slice, D.E., Stitzel, J.D., 2006. A finite element study of age-based size and 22 shape variation of the human rib cage. Biomedical sciences instrumentation 42, 19-24.

23 Gayzik, F.S., Yu, M.M., Danelson, K.A., Slice, D.E., Stitzel, J.D., 2008. Quantification of age-related 24 shape change of the human rib cage through geometric morphometrics. Journal of biomechanics 41, $25 \quad 1545-1554$. 
1 Hayashi, S., Yasuki, T., Kitagawa, Y., 2008. Occupant kinematics and estimated effectiveness of side

2 airbags in pole side impacts using a human FE model with internal organs. Stapp car crash journal 52,

3363.

4 Ito, O., Dokko, Y., Ohashi, K., 2009. Development of adult and elderly FE thorax skeletal models. SAE

5 Technical Paper.

6 Iwamoto, M., Kisanuki, Y., Watanabe, I., Furusu, K., Miki, K., Hasegawa, J., 2002. Development of a

7 finite element model of the total human model for safety (THUMS) and application to injury

8 reconstruction. In Proceedings of the International Research Council on the Biomechanics of Injury

9 conference.

10 Kent, R., Lee, S.-H., Darvish, K., Wang, S., Poster, C.S., Lange, A.W., Brede, C., Lange, D., Matsuoka,

11 F., 2005. Structural and material changes in the aging thorax and their role in crash protection for older

12 occupants. Stapp car crash journal 49, 231.

13 Kimpara, H., Lee, J.B., Yang, K.H., King, A.I., Iwamoto, M., Watanabe, I., Miki, K., 2005. Development

14 of a three-dimensional finite element chest model for the 5th percentile female. Stapp car crash journal 49, 15251.

16 Kindig, M.W., Lau, A.G., Forman, J.L., Kent, R.W., 2010. Structural response of cadaveric ribcages

17 under a localized loading: stiffness and kinematic trends. Stapp car crash journal 54, 337.

18 Klein, K.F., Hu, J., Reed, M.P., Hoff, C.N., Rupp, J.D., 2015. Development and Validation of Statistical

19 Models of Femur Geometry for Use with Parametric Finite Element Models. Annals of biomedical

20 engineering 43(10), 2503-2514.

21 Lamecker, H., Lange, T., Seebass, M., 2004. Segmentation of the liver using a 3D statistical shape model.

22 Konrad-Zuse-Zentrum für Informationstechnik.

23 Lu, Y.-C., Untaroiu, C.D., 2014. A statistical geometrical description of the human liver for probabilistic 24 occupant models. Journal of biomechanics 47, 3681-3688. 

Ma, X., Laud, P.W., Pintar, F., Kim, J.-E., Shih, A., Shen, W., Heymsfield, S.B., Allison, D.B., Zhu, S., 2011. Obesity and non-fatal motor vehicle crash injuries: sex difference effects. International Journal of

3 Obesity $35,1216-1224$.

4 Parenteau, C.S., Zuby, D., Brolin, K., Svensson, M.Y., Palmertz, C., Wang, S.C., 2013. Restrained Male 5 and Female Occupants in Frontal Crashes: Are We Different? In 2013 IRCOBI Conference Proceedings.

6 Ridella, S.A., Rupp, J.D., Poland, K., 2012. Age-related differences in AIS 3+ crash injury risk, types,

7 causation and mechanisms. In Proceedings of the International Research Council on the Biomechanics of 8 Injury conference.

9 Shi, X., Cao, L., Reed, M.P., Rupp, J.D., Hoff, C.N., Hu, J., 2014. A statistical human rib cage geometry 10 model accounting for variations by age, sex, stature and body mass index. Journal of biomechanics 47 , $11 \quad 2277-2285$.

12 Shi, X., Cao, L., Reed, M.P., Rupp, J.D., Hu, J., 2015. Effects of obesity on occupant responses in frontal 13 crashes: a simulation analysis using human body models. Computer methods in biomechanics and 14 biomedical engineering 18, 1280-1292.

15 Slice, D.E., 2006. Modern morphometrics in physical anthropology. Springer Science \& Business Media. 16 Stegmann, M.B., Gomez, D.D., 2002. A brief introduction to statistical shape analysis. Informatics and 17 Mathematical Modelling, Technical University of Denmark, DTU 15, 11.

18 Turkovich, M.J., 2011. The effects of obesity on occupant injury risk in frontal impact: a computer 19 modeling approach. PhD. thesis, University of Pittsburgh.

20 Wang, Y., Bai, Z., Cao, L., Reed, M.P., Fischer, K., Adler, A., Hu, J., 2015. A simulation study on the 21 efficacy of advanced belt restraints to mitigate the effects of obesity for rear-seat occupant protection in 22 frontal crashes. Traffic injury prevention 16, S75-S83.

23 Weaver, A.A., Schoell, S.L., Nguyen, C.M., Lynch, S.K., Stitzel, J.D., 2014a. Morphometric analysis of 24 variation in the sternum with sex and age. Journal of morphology 275, 1284-1299.

25 Weaver, A.A., Schoell, S.L., Stitzel, J.D., 2014b. Morphometric analysis of variation in the ribs with age 26 and sex. Journal of anatomy 225, 246-261. 
1 Yates, K.M., Lu, Y.-C., Untaroiu, C.D., 2016. Statistical shape analysis of the human spleen geometry for 2 probabilistic occupant models. Journal of biomechanics 49, 1540-1546.

3 Zhou, Q., Rouhana, S.W., Melvin, J.W., 1996. Age effects on thoracic injury tolerance. In Proceedings:

$4 \quad$ Stapp Car Crash Conference 40, 137-148.

5 Zhu, Z., Li, G., 2011. Construction of 3D human distal femoral surface models using a 3D statistical 6 deformable model. Journal of biomechanics 44, 2362-2368. 

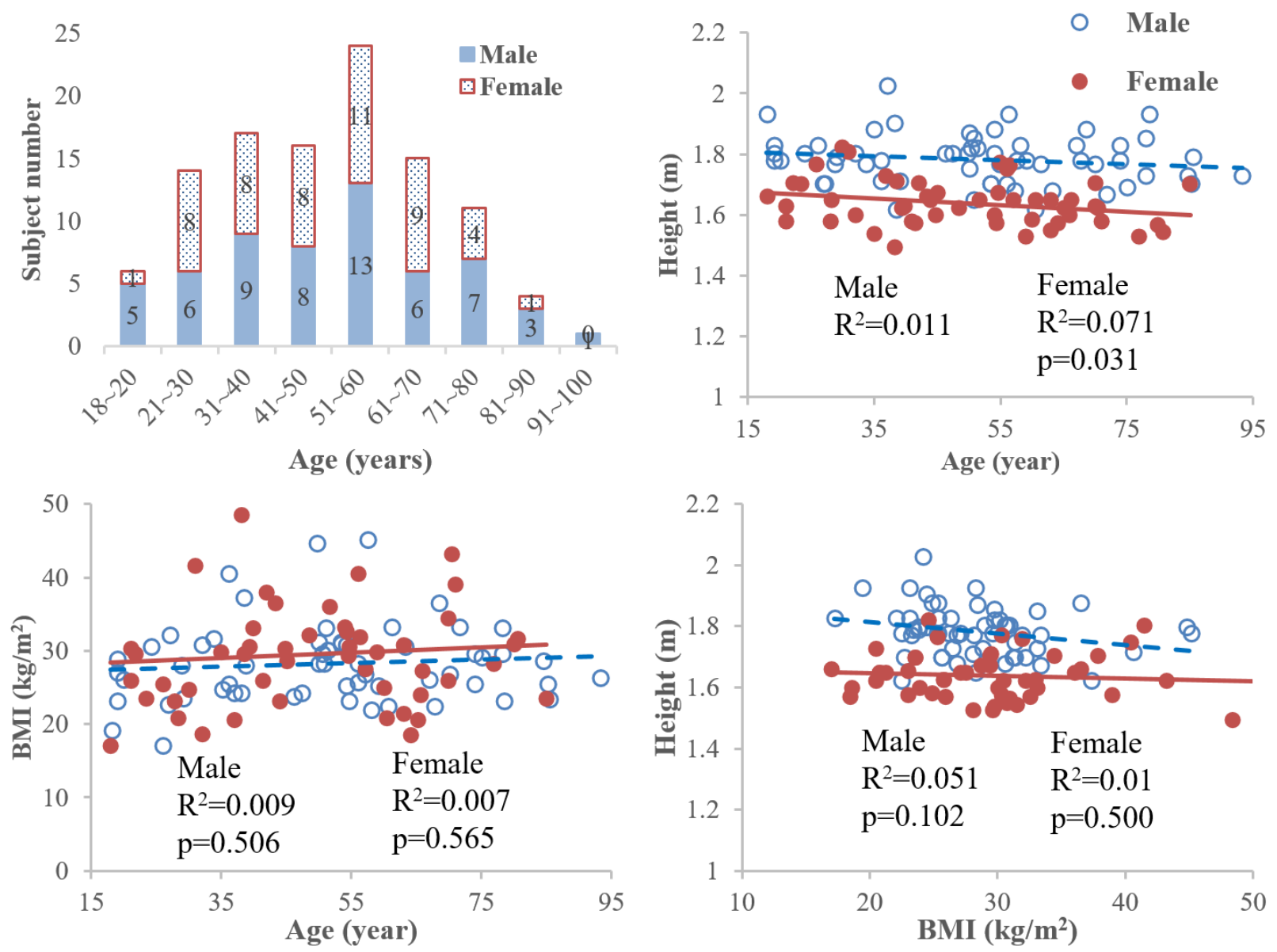

Figure 1. Age, sex, height and BMI distributions of all sampled subjects 


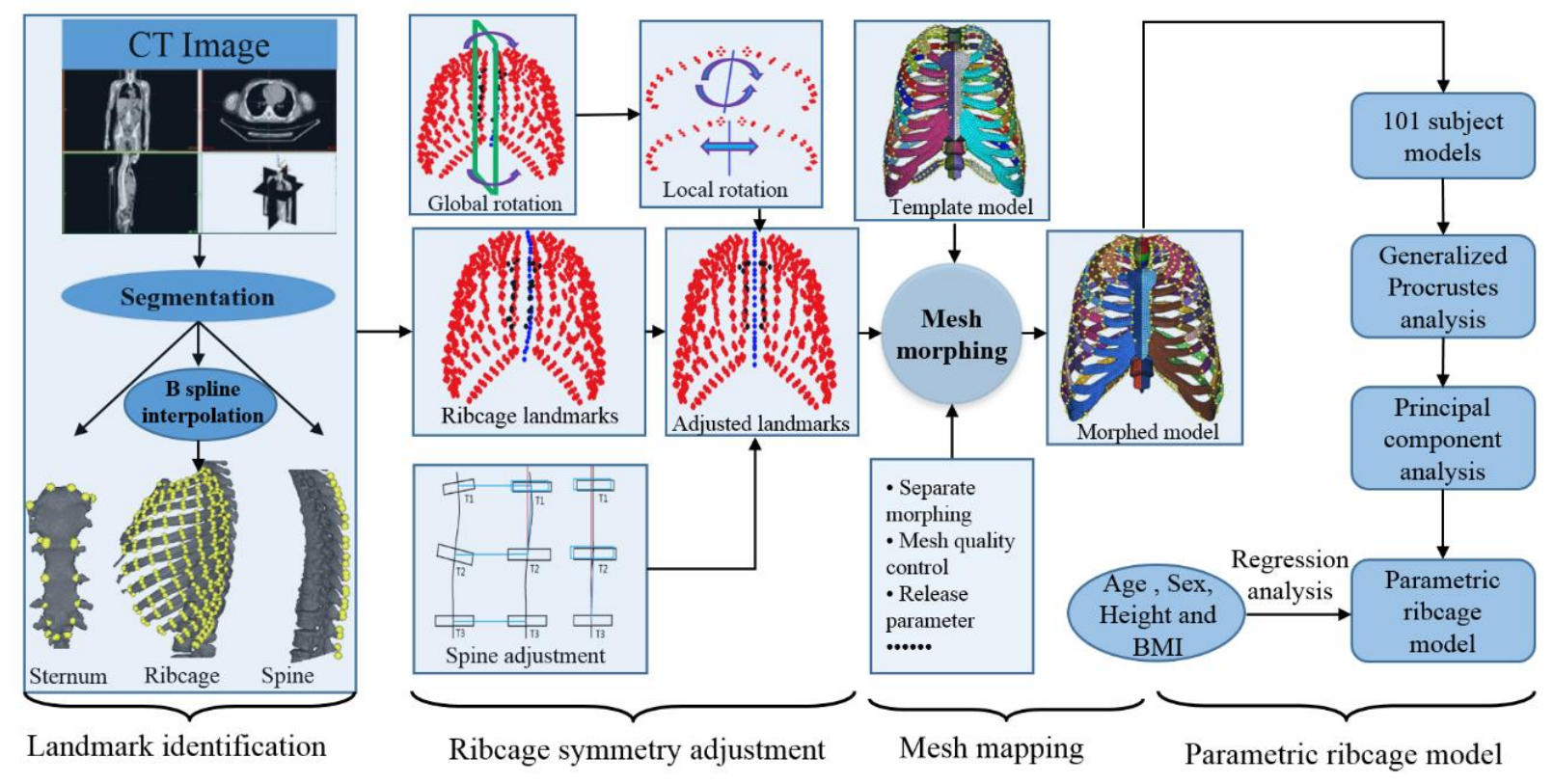

Figure 2. Method overview for developing parametric ribcage model 

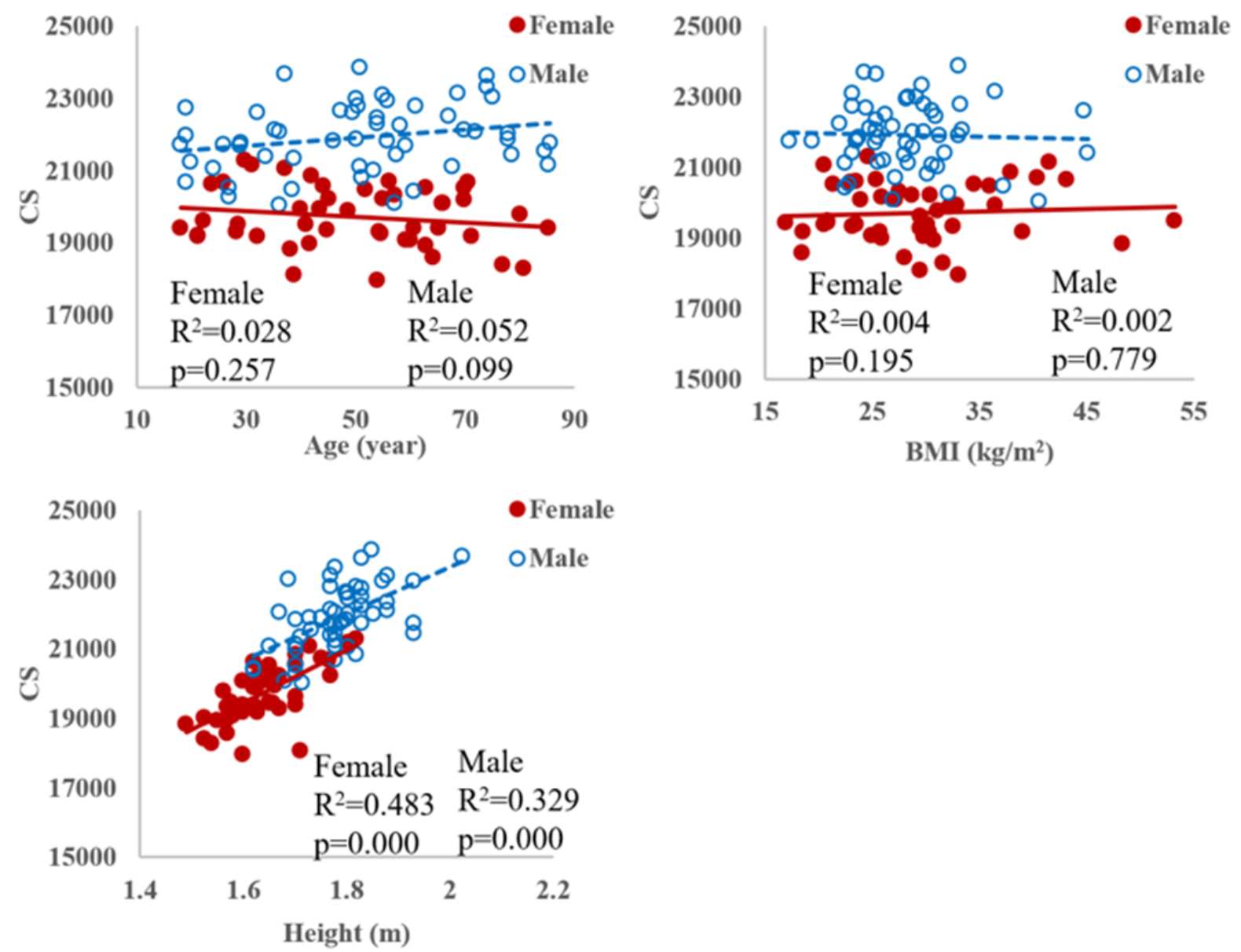

CS is the centroid size shown in Equation 3.

Figure 3. Age, sex, height and BMI effects on the centroid size of ribcage 


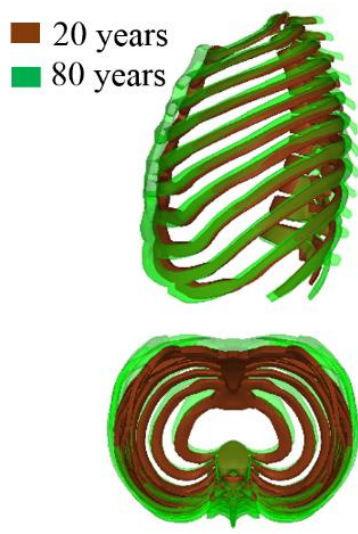

BMI: $25 \mathrm{~kg} / \mathrm{m}^{2}$

Height: $1.75 \mathrm{~m}$

Sex: male

(a) Age effect

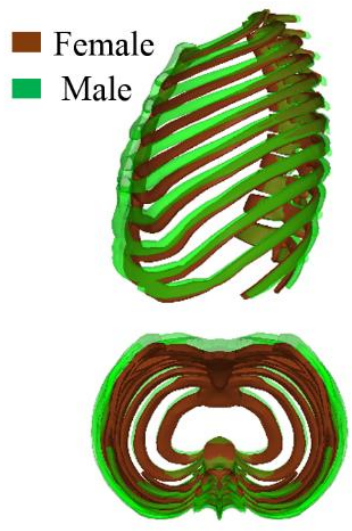

Age: 35 years

BMI: $25 \mathrm{~kg} / \mathrm{m}^{2}$

Height $1.75 \mathrm{~m}$

(b) Sex effect

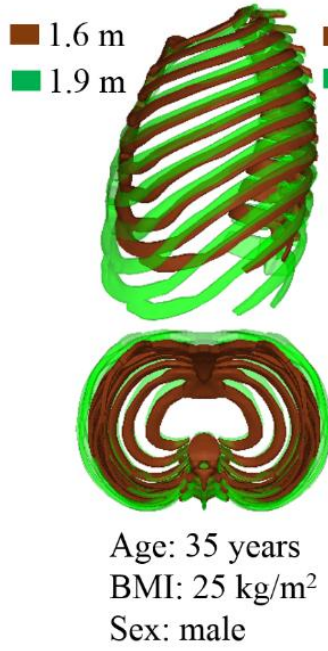

(c) Height effect
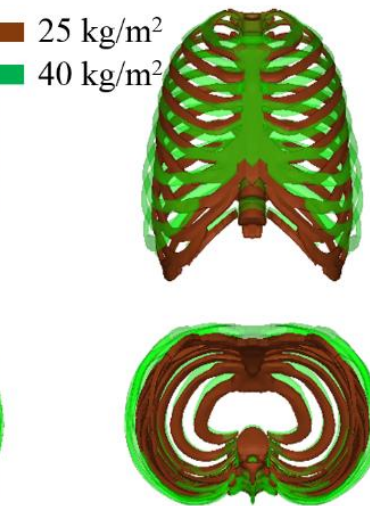

Age: 35 years

Height $1.75 \mathrm{~m}$

Sex: male

(d) BMI effect

Figure 4. Age, sex, height, and BMI effects on ribcage geometry 

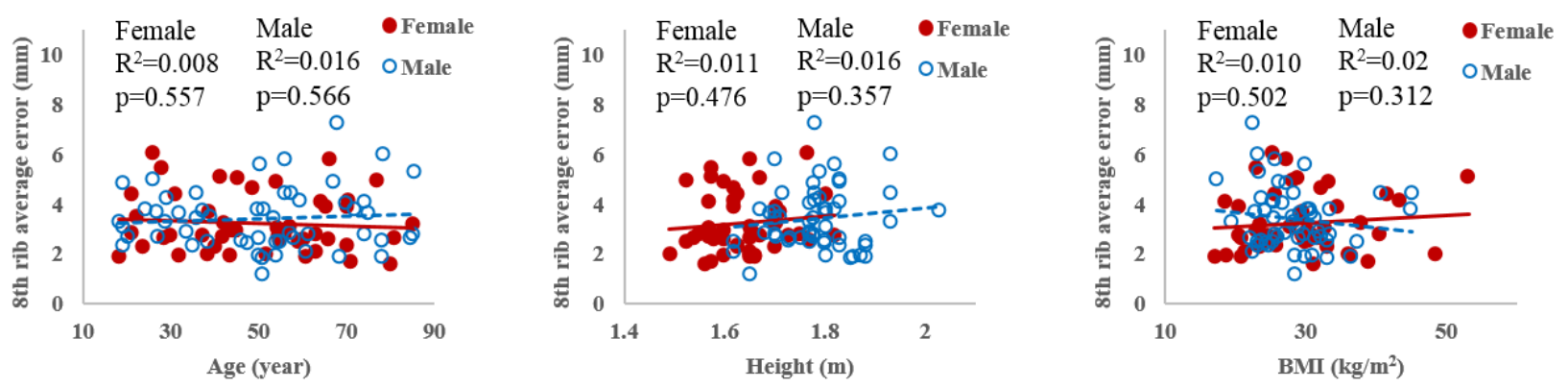

(a) Average errors of the $8^{\text {th }}$ rib
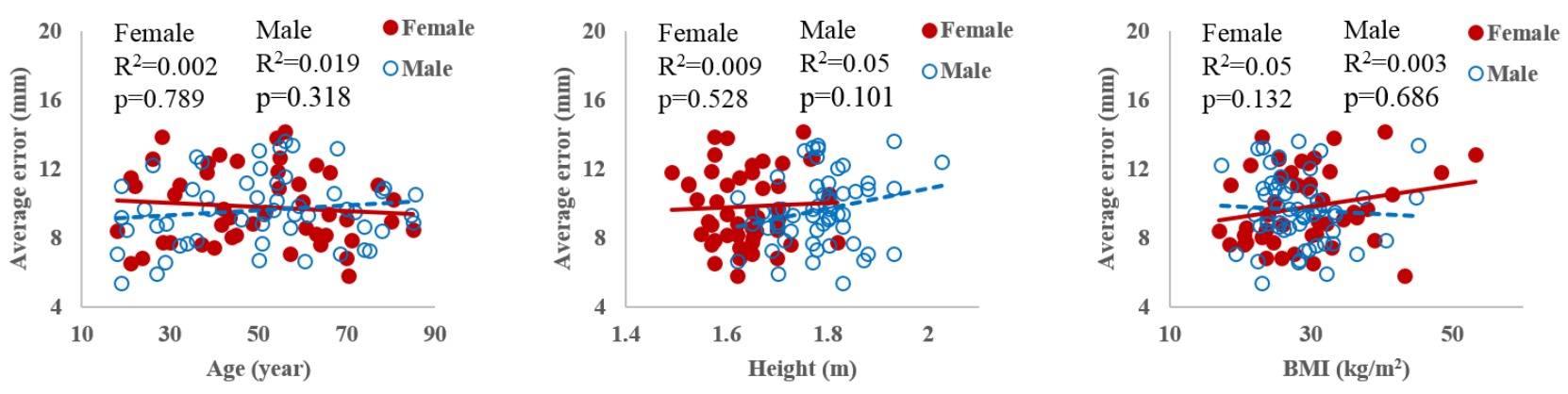

(b) Average errors of the whole ribcage

Figure 5. Average errors a single rib and whole ribcage with respect to age, height, and BMI 


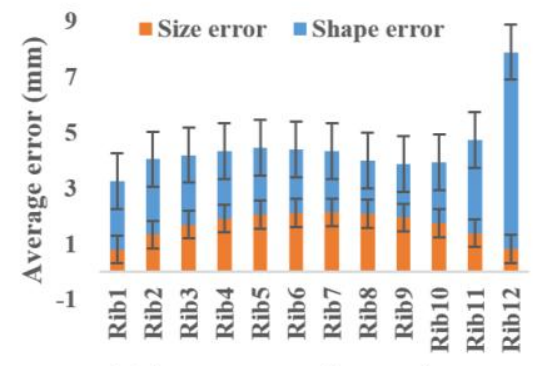

(a) Average error distance for the single rib

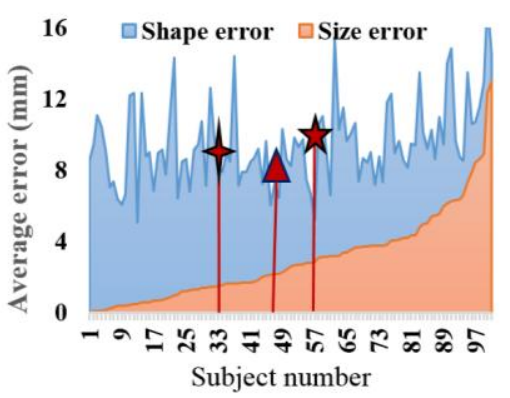

(b) Average error distance for the whole ribcage

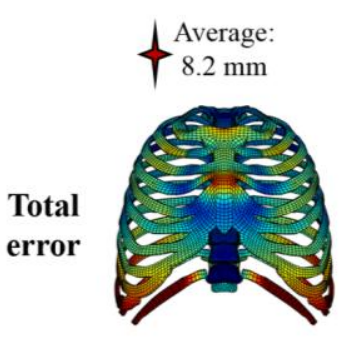

Average:

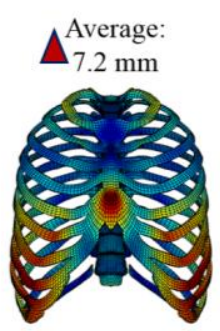

Average:

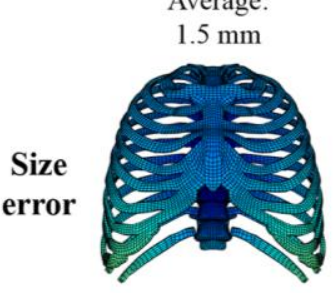

Age: 38 years

BMI: $48 \mathrm{~kg} / \mathrm{m}^{2}$

Height: $1.49 \mathrm{~m}$

Sex: Female

\# FG3S105

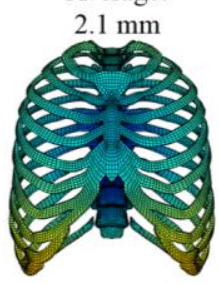

Age: 29 years

BMI: $28 \mathrm{~kg} / \mathrm{m}^{2}$

Height: $1.77 \mathrm{~m}$

Sex: Male

\# MG2S107

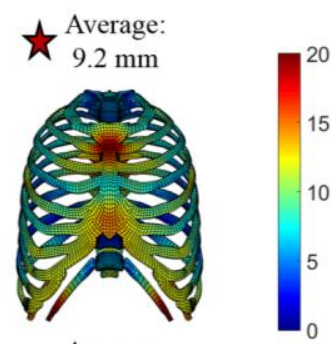

Average:

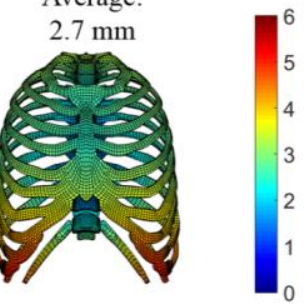

Age: 50 years

BMI: $28 \mathrm{~kg} / \mathrm{m}^{2}$ Height: $1.87 \mathrm{~m}$ Sex: Male

\# MG5S119

(c) Error distributions for three different subjects

Figure 6. Error distance caused by size regression model and shape regression model 

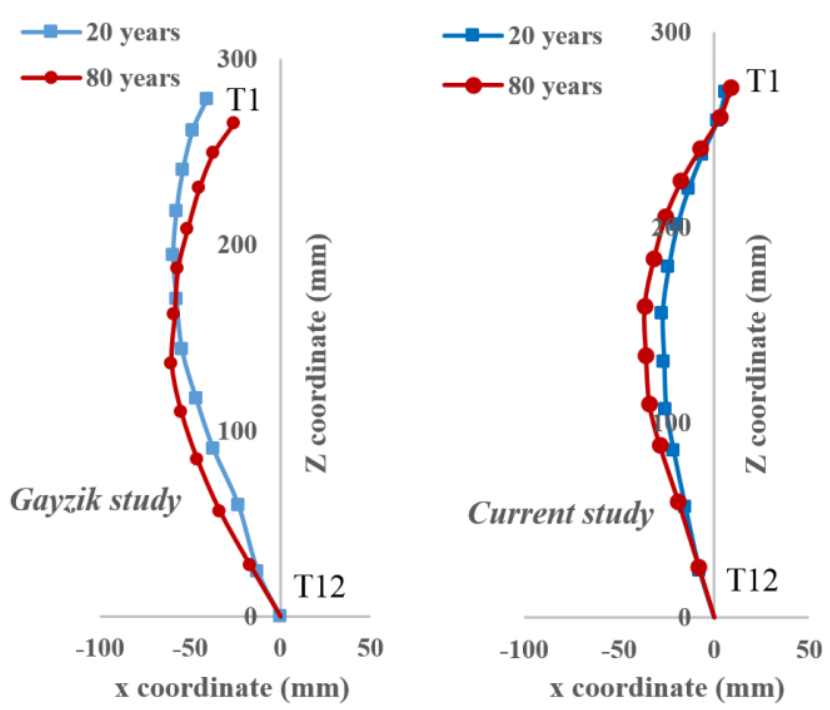

Figure 7. Age effect on thoracic spine curvature for men 